\title{
How can pediatricians treat neurodevelopmental disorders
}

\author{
Young-Hoon Kim, MD, PhD \\ Department of Pediatrics, College of Medicine, The Catholic University of Korea, Seoul, Korea
}

\begin{abstract}
Key message
- Recently neurodevelopmental therapy for preschool-aged children with neurodevelopmental disorders is paid for by health insurance in Korea.

- There are good evidences that parenting programs and neurodevelopmental therapy can work in attention deficit hyperactivity disorder and autism spectrum disorder.

- Pediatricians must be able to pass away important information to parents.
\end{abstract}

Although neurodevelopmental therapy for preschool-aged children with neurodevelopmental disorders recently became reimbursable by health insurance in Korea, there is a little of evidence relating to the effectiveness of parenting programs and neurodevelopmental therapy for children with attention deficit hyperactivity disorder (ADHD) or autism spectrum disorder (ASD).

A review of parenting programs found them ineffective for hyperactivity or impulsivity but somewhat helpful for improving attention. Neurodevelopmental therapy had stronger effects than medications managed in the pediatric clinic. Psychological therapies and multimodal interventions had greater effects than parenting programs. However, parenting programs had the largest effect for general cognition. ${ }^{1)}$ There is insufficient evidence to recommend treatment for children younger than 4 years other than parenting programs and neurodevelopmental therapy in behavior management. For preschool-aged children (age 4-6 years) with ADHD, the pediatricians should prescribe parenting program and neurodevelopmental therapy as the first line of treatment, if available. ${ }^{2)}$ Medication may be considered if this approach does not result in significant improvement and there is moderate to severe continued disturbance in the 4- to 5 -year-old child's functioning. ${ }^{2)}$ In areas in which parenting program and neurodevelopmental therapy are not available, the pediatricians need to evaluate the risks of starting medication before the age of 6 years against the harm of delaying treatment. A number of special circumstances support the recommendation to initiate parenting programs and neurodevelopmental therapy as the first treatment of preschool-aged children (4-6 years of age).

Parenting programs and neurodevelopmental therapy are dyadic therapies for parent and child. ${ }^{3)}$ Preschool programs can also provide behavioral supports. In areas in which evidencebased behavioral treatments are not available, the pediatrician needs to evaluate the risks of starting methylphenidate before the age of 6 years against the harm of delaying diagnosis and treatment. Other stimulant or nonstimulant medications have not been adequately studied in children in this age group with ADHD. ${ }^{2)}$

The evidence regarding parenting programs for children with ASD is controversial. ${ }^{4)}$ Autism preschool parenting programs and neurodevelopmental therapy had nonsignificant improvements in cognitive measures, children's communication and levels of disruptive behavior, parental knowledge and mother-child interaction.5) Parenting programs and neurodevelopmental therapy significantly reduced ratings on the Autism Diagnostic Observation Schedule. Those results showed significant effects of parenting programs and neurodevelopmental therapy on receptive languages and expressive languages. Social communication parenting programs led to significantly greater interaction approaches with their child involving greater parental synchrony. Observations of happiness, interest, low stress and communication style were rated better in parenting programs groups. ${ }^{4)}$

There is good evidence that parenting programs and neurodevelopmental therapy can be effective for children with ADHD and ASD (Table 1). Parenting programs vary considerably in their theoretical background. Some approaches utilize applied behavior analysis in intensive programs based at home, but it is

Table 1. The pediatrician should initiate an evaluation for preschool-aged children with ADHD or ASD

\begin{tabular}{lll}
\hline Aggregate evidence & \multicolumn{1}{c}{ ADHD } & ASD \\
\hline Benets & The bene ts outweigh the risks. & Effective on cognition, language, and social communication \\
Risks, harm, cost & A high level of family involvement & The major risk is providing inappropriate care. \\
\hline
\end{tabular}

$\mathrm{ADHD}$, attention deficit hyperactivity disorder; ASD, autism spectrum disorder.

Corresponding author: Young-Hoon Kim, MD, PhD. Department of Pediatrics, Uijeongbu St. Mary's Hospital, Catholic University School of Medicine, 271 Cheonbo-ro, Uijeongbu 11765, Korea 
important to recognize that there is no parenting program and neurodevelopmental therapy which can be universally appropriate for all children and parents. Thus, pediatricians should choose notable parenting programs in ADHD and ASD. The findings of the 2 neurodevelopmental disorders groups reveal 2 key implications for parenting policy. Parenting programs and neurodevelopmental therapy should consist of a more organized and individual-centered approach. A more organized service can be provided if there is consistency in the sharing of information between pediatricians and parents using a clear structure.

Pediatricians must be able to disseminate important information to parents to ensure that they are aware of the parenting programs and neurodevelopmental therapies are available to them.

\section{Conflicts of interest}

No potential conflicts of interest relevant to this article are reported.

\section{References}

1. Evans SW, Owens JS, Wymbs BT, Ray AR. Evidence-based psychosocial treatments for children and adolescents with attention deficit/hyperactivity disorder. J Clin Child Adolesc Psychol 2018;47:157-98.

2. Wolraich ML, Hagan JF Jr, Allan C, Chan E, Davison D, Earls M, et al. Clinical practice guideline for the diagnosis, evaluation, and treatment of attention-deficit/hyperactivity disorder in children and adolescents. Pediatrics 2019;144:e20192528.

3. Law, J, Plunkett C, Taylor J, Gunning M. Developing policy in the pro vision of parenting programmes: integrating a review of reviews with the perspectives of both parents and professionals. Child Care Health Dep 2009;35:302-12

4. Diggle T, McConachie HR, Randle VR. Parent-mediated early intervention for young children with autism spectrum disorder. Cochrane Database Syst Rev 2003:(1);CD003496.

5. McConachie H, Diggle T. Parent implemented early intervention for young children with autism spectrum disorder: a systematic review. J Eval Clin Pract 2007:13;120-9.

How to cite this article: Kim YH. How can pediatricians treat neurodevelopmental disorders. Clin Exp Pediatr 2021;64:1-2. https://doi.org/10.3345/cep.2020.00507 